\title{
„Ich war lange illegal hier, aber jetzt hat mich die Grenze übertreten"-Subjektivierungsprozesse transnational mobiler Haushaltshilfen
}

\author{
A. Strüver \\ Institut für Geographie, Universität Hamburg, Hamburg, Germany \\ Correspondence to: A. Strüver (struever@geowiss.uni-hamburg.de)
}

Received: 31 July 2012 - Revised: 25 February 2013 - Accepted: 25 February 2013 - Published: 7 October 2013

Zusammenfassung. This contribution examines the processes of subjectivation of transnational mobile female migrants from Eastern Europe, who work irregularly as domestic workers in Germany. Applying an intersectional framework, the working practices of female migrants are conceptualized as an expression of the interplay between technologies of the self and technologies of domination. Both are constitutive for the migrant subjects and they are thus analyzed as part of geopolitical constellations and economic power relations as well as being influenced by the European border and labor market regime.

\section{Einleitung}

Viele migrantische Haushaltshilfen in Deutschland führen ein transnational mobiles Arbeitsleben. Sie streben damit keine klassische Einwanderung an, sondern eine anhaltende Pendelmigration, die kurz- oder mittelfristig eine Alternative zu ökonomisch prekären Beschäftigungen ,zuhause“ darstellt. Insbesondere als so genannte Live-In-Haushaltshilfen arbeiten die meisten von ihnen als Teil eines „Duos“, d.h. sie übernehmen eine paarweise angelegte Rund-um-die-UhrBetreuung $\left(, 24 / 7^{\circ}\right)$ von alten oder kranken Menschen und führen teilweise auch deren Haushalt. Ein solches Duo wechselt sich allerdings nicht in Tag- und Nachtschichten o.ä. ab, sondern wöchentlich, monatlich oder längerfristig in einem selbstorganisierten Rotationsprinzip.

Dieser Beitrag untersucht die politischen, sozioökonomischen und vor allem subjektiven Dimensionen dieser Art von grenzüberschreitender Mobilität. Dabei stehen die Arbeitsund Alltagspraktiken von Migrantinnen im Vordergrund, an denen sich die gesellschaftliche Konstitution von Subjektivierungsprozessen als Teil geopolitischer und -ökonomischer Machtformationen sowie europäischer Grenz- und Arbeitsmarktregime zeigt.

\section{Gesellschaftliche Transformationen in Deutschland und Europa}

Die politischen, gesellschaftlichen und rechtlichen Rahmenbedingungen für die steigende Zahl von transnational agierenden Haushaltshilfen in Deutschland liegen einerseits in der wachsenden Nachfrage an Dienstleistungen in Privathaushalten, insbesondere zur Versorgung von Kindern und Pflegebedürftigen oder auch als so genannte Putzkraft. Der Bedarf an Migrantinnen in diesen Bereichen wird als ,spätmoderner Dienstmädchen-Service“ (Römhild, 2007:218) beschrieben und basiert im Wesentlichen auf den Auswirkungen des demographischen Wandels, vor allem der steigenden Lebenserwartung und der damit einhergehenden Zunahme an Pflegebedürftigen, bei zeitgleicher Abnahme des Erwerbspersonenpotenzials im Allgemeinen und des Pflegepersonals im Besonderen (für Prognosen bis 2020, siehe Pohl, 2010). Dementsprechend ist im Bereich der häuslichen Pflege älterer und/oder kranker Menschen der Anteil an irregulär arbeitenden Migrantinnen, die diese Versorgung ,günstig und flexibel" übernehmen, in Deutschland stark anwachsend. Zugleich stellen Privathaushalte den wichtigsten irregulären Arbeitgeber für transnational pendelnde migrantische Haushaltshilfen dar und es ist ein transnationales Migrations- und Arbeitsregime beobachtbar, ,das sich informell und nichtsdestoweniger in der Öffentlichkeit entwickelte“ (Karakayali, 
2010:17). Die Migrantinnen bedienen folglich den Bedarf an familienähnlichen Reproduktionsdienstleistungen und können durch das Rotationsprinzip im Duo zugleich regelmäßig „,nach Hause" pendeln. ${ }^{1}$

Andererseits beruht die transnationale Migration von weiblichen Haushaltshilfen im innereuropäischen Kontext auf spezifischen Voraussetzungen und Ausgestaltungen, da sich im Zuge der letzten zwei EU-Erweiterungen die Aufenthalts- und Arbeitsrechte für Migrant_innen mehrmals geändert haben: So verfügten Migrant_innen aus den Staaten der EU-Osterweiterung von 2004 in Deutschland bis Mai 2011 zwar über eine Aufenthaltserlaubnis, nicht aber über eine Arbeitserlaubnis (für die Beitrittsländer aus dem Jahr 2007, Rumänien und Bulgarien, gilt diese „Beschränkung der Arbeitnehmerfreizügigkeit" noch bis Ende 2013). D.h. für die Zeit vor Mai 2011 (bzw. bis Januar 2014) ließ (lässt) sich feststellen, dass sich diese EU-Migrant_innen zwar legal in Deutschland aufhalten, aber (wie auch Angehörige aus den ost- und südosteuropäischen Nicht-EUStaaten) in der Regel nur irregulär einer Arbeit nachgehen können. ${ }^{2}$ Doch trotz mittlerweile erfolgter Öffnung des regulären deutschen Arbeitsmarktes belegen erste Bilanzen seit Mai 2011 im Bereich der haushaltsnahen Dienstleistungen die Tendenz eines Verbleibens in der Irregularität, da sich dies für die Arbeitgeber_innen, vor allem aber auch für die Migrant_innen als finanziell profitabler sowie als flexibler gestaltbar erweist (vgl. Baas und Brücker, 2011). ${ }^{3}$ Der letztgenannte Punkt wiederum verweist auch auf das Potenzial, das viele der osteuropäischen migrantischen Haushaltshilfen in ihrer Arbeit in deutschen Privathaushalten sehen: Sie schließt, zumindest vorübergehend, die Versorgungslücken, die in ihren Herkunftsländern in Folge der soziökonomischen Transformationen entstanden sind. Besonders deutlich wird dies anhand der Feststellung, dass zwar viele der osteuropäischen Reinigungs- und Betreuungskräfte in deutschen Privathaushalten über eine abgeschlossene Berufs- oder Universitätsausbildung verfügen, mit der Qualifikation als Lehrerin, Krankenschwester, Sozialpädagogin oder Juristin jedoch kei-

\footnotetext{
${ }^{1}$ Nach Schätzungen des Statistischen Bundesamtes (2009) arbeiten ca. drei Millionen irregulär Beschäftige in deutschen Haushalten; ca. ein Drittel davon sind irreguläre Migrant_innen - von denen ca. 120.000 als Live-In-Pflegekräfte arbeiten.

${ }^{2}$ Darüber hinaus fanden auch bereits vor den Osterweiterungen Migrationsbewegungen aus den Beitrittskandidatenländern nach Deutschland statt; je nach Status im Herkunftsland meist mit Touristen- oder Studierendenvisa.

${ }^{3}$ Dies gilt sowohl für unqualifizierte (bzw. beruflich anders qualifizierte) als auch für in Pflegeberufen qualifizierte Osteuropäerinnen; von Letzteren wird die Irregularität bevorzugt, da ihre Ausbildungen in Deutschland bislang nicht anerkannt sind, so dass ihre Entlohnung höher ist, wenn sie als irregulär Beschäftigte tätig sind. Flexible Arbeitsarrangements umfassen hier zum einen zeitliche Absprachen; zum anderen allerdings auch fehlende Rechtsansprüche im Fall von Krankheit, Kündigung oder bei Nichteinhaltung von Zahlungsabsprachen.
}

ne Anstellung finden können bzw. damit keine angemessene Entlohnung erhalten (vgl. die zahlreichen Beispiele in Karakayali, 2010; Lutz, 2008; Metz-Göckel, 2010b und Rerrich, 2006). Während die Chancen auf eine der Ausbildung angemessene Beschäftigung und Bezahlung gerade für junge Universitätsabsolventinnen in vielen osteuropäischen Staaten eher gering sind, zählt als Haushaltshilfe in Deutschland nicht ihr Abschluss, sondern die an das Geschlecht gekoppelten Assoziationen von „Haus-Frau“-Sein. Die Beschäftigung von weiblichen migrantischen Haushaltshilfen basiert somit auf den dominanten Assoziationen von geschlechtlich codierten Arbeits- und Lebensbereichen und reproduziert diese auch in hohem Maße. Sie basiert gleichwohl neben dem „Frau-Sein“ und in bedeutsamem Umfang auch auf der fehlenden Aufenthalts- und/oder Arbeitserlaubnis, d.h. auf ihrem Ausländerin-Sein oder sogar Illegalisiert-Sein. ${ }^{4}$ Denn die Mobilität migrantischer Haushaltshilfen wird neben der vermeintlich individuell oder familiär getroffenen Entscheidung durch die zwischen einzelnen Staaten etablierten Grenz- und Migrationsregime reguliert: Sozioökonomische Disparitäten führen zu unterschiedlichen Wertigkeiten beruflicher Qualifikationen, aber auch sozialer Fähigkeiten wie professionalisierter Emotionen. Die emotionalen und sozialen Kompetenzen von Migrantinnen sowie ihr Frau-Sein und ihr Illegalisiert-Sein werden in Form sozialstruktureller Kategorisierungen qua Geschlecht, Alter und Nationalität zum Vor- oder Nachteil eines Subjekts - und variieren in ihrer Bedeutung je nach nationalem Kontext: „Dies ist zum Beispiel bei osteuropäischen Akademikerinnen der Fall, die in deutschen Haushalten putzen. Aus deutscher Sicht ist ihr kulturelles Kapital entwertet, ihnen fehlt die Arbeitserlaubnis (...). Im polnischen Bezugsmaßstab handelt es sich um relativ gut verdienende sozial [und räumlich] mobile Unternehmerinnen“"(Weiß, 2010:367).

Die Entwertung des institutionalisierten kulturellen Kapitals, d.h. eines Berufs- oder Universitätsabschlusses, durch fehlende Aufenthalts- und/oder Arbeitsgenehmigungen wird in der klassischen Migrationsforschung auch als „Brain Drain" bezeichnet und bezieht sich neben der Dequalifikation von Migrant_innen vorrangig auf die Verluste für die Herkunftsgesellschaften. Bauder (2003) geht noch einen Schritt weiter und spricht in diesem Zusammenhang von „Brain Abuse“, da die Zielregionen zwar von der niedrigen Entlohnung der als Unqualifizierte eingestuften Migrant_innen profitieren, aber zugleich das inkorporierte Humankapital ungenutzt lassen. Er zitiert dafür die Kritik eines kanadischen Arbeitsmarktexperten an dieser Art der Migrationspolitik (fehlende

\footnotetext{
${ }^{4}$ Informelle Aufenthalte sowie irreguläre Arbeitsverhältnisse von Migrant_innen werden oftmals unter ,Illegalität“" subsumiert, so dass vielerorts von „Illegalen“ gesprochen wird. Neben der Tatsache, dass ,,informell“ und ,,irregulär“ nicht mit ,illegal“ gleichzusetzen sind, sollte eher von Illegalisierten gesprochen werden, da Menschen an sich nicht legal oder illegal sein können. Vielmehr wird die Kategorie der Illegalität erst durch politische und juristische Beschlüsse produziert.
} 
Aufenthalts- und Arbeitsgenehmigungen bzw. keine Anerkennung von im Ausland erworbenen Berufsqualifikationen): „That country from where the person has immigrated loses that brain, this country who should have been otherwise benefited from that quality doesn't accept that brain. (...) It is a brain abuse because it's not proper utilization of the quality and the skills of people" (Bauder, 2003:714f). Aus der Perspektive vieler der migrantischen Haushalts- und Pflegekräfte in Deutschland, die unabhängig von ihrer beruflichen Qualifikation arbeiten, liest sich dieser „Brain Abuse“ jedoch auch anders: Sie haben als irregulär Beschäftigte ein vergleichsweises hohes Einkommen. Die Pendelmigration wird dabei zunächst als temporäre Lebensform gewählt, um die materielle Situation zu verbessern. Die Bereitschaft zur anhaltenden Mobilität spielt dafür eine besonders wichtige Rolle - und ist nicht als explizit widerständige, wohl aber als eigenständige (,selbst-ständige“) Praktik zu verstehen. Denn mit der grenzüberschreitenden räumlichen Mobilität geht in den meisten Fällen anfänglich eine soziale Abwärtsmobilität einher, die sich gleichwohl ökonomisch als profitabel erweist.

\section{3 „Erst war alles provisorisch, dann für Provision!“}

Um die Subjektivierungsprozesse transnational mobiler Haushaltshilfen nachvollziehbar zu machen, wird hier aus insgesamt 28 narrativ-biographischen Interviews mit jungen Frauen aus Polen, der Slowakei, Litauen, Rumänien und Bulgarien (EU-Beitrittsländer von 2004 bzw. 2007) sowie der Ukraine und Weißrussland (keine EU-Mitglieder) zitiert. Sie alle verfügen über eine qualifizierte Berufs- oder Universitätsausbildung, sind aber aufgrund der als zu eingeschränkt wahrgenommenen Lebens- und Karriereperspektiven selbstorganisiert als Haushaltshilfen nach Deutschland gekommen. Sie sind größtenteils länger geblieben als ursprünglich geplant und bewegen sich in einem transnationalen Rotationsgefüge, das zeitlich wie räumlich auf unterschiedlich angelegten Zyklen basiert. $^{5}$

Maria ist zum Zeitpunkt des Interviews (September 2010) bereits 40 Jahre alt und pendelt seit 1994 regelmäßig zwischen Breslau und Hamburg. Anlass für ihre erste Reise, die sie mit einem Touristenvisum antrat, war die akute Pflegebedürftigkeit einer Großtante in Hamburg. Obwohl sie gerade

\footnotetext{
${ }^{5}$ Die Interviews mit den als transnationale Haushaltshilfen arbeitenden Migrantinnen (anonymisierte Namen) wurden zwischen 2009 und 2012 in verschiedenen norddeutschen Städten geführt. Die Auswahlkriterien für die Interviewpartnerinnen waren ihr ,,junges Alter" (zwischen 20 und 30 Jahren), ein qualifizierender Berufsoder Universitätsabschluss sowie Kinderlosigkeit (zum Zeitpunkt des Arbeitsbeginns in Deutschland).

Im hier vorliegenden Kontext dienen die Interviews vor allem der Rekonstruktion ,,intersektionaler Subjektpositionen in der Krise“, d.h. einer Analyse des Zusammenwirkens verschiedener Identitätskategorien sowie deren Veränderungen durch das grenzüberschreitende Pendeln.
}

frisch die Universität mit einem Examen in Jura abgeschlossen hatte, ,stand mir der Sinn nicht nach weiter arbeiten ... also weiter qualifizieren und studieren, um direkt Richter zu werden oder so. Ganz im Gegenteil, diese innerfamiliäre Krisensituation, da ist die Tante Hala, um die sich jemand kümmern muss, aber keinen dafür bezahlen will ... das war für mich eine Herausforderung, oder eigentlich: der Reiz vom Neuen, vom Anderen und vor allem, dass ich dafür nach Deutschland ... nicht nur durfte, sondern quasi musste.“

Während der insgesamt fast vierjährigen Pflegezeit bis zum Tod der Tante lernt Maria aufgrund von nur sehr begrenzten außerhäuslichen Kontakten im ersten Jahr kaum Deutsch, pendelt aber regelmäßig für kurze Zeit nach Breslau, um ein neues Visum zu beantragen. „Dann gab es einmal diesen Zwangsaufenthalt zuhause [gemeint ist Breslau] wegen der neuen Visa, also da ging fast sechs Monate nichts und wir hatten in Hamburg bereits eine andere Cousine für die Pflege organisiert. Also diese Pause, da habe ich schon überlegt, was ich denn eigentlich will und mir war klar, dass das nicht Füttern, Waschen, Putzen, Warten, warten, warten und immer wieder warten ist! Während des vielen Wartens [im Haushalt der Tante] hatte ich so viel Fernsehen geguckt, dass mein Deutsch dann ganz gut war, aber damit konnte ich wenig anfangen, da ich ja kaum jemand Deutsches kannte und offiziell natürlich gar nicht da war, also auch nicht woanders arbeiten konnte." Sobald sie ein neues Visum bekommt, fährt sie dann aber doch direkt wieder nach Hamburg, mit dem Vorsatz, sich dort um ein Aufbaustudium zu kümmern. Da ihre Cousine sie allerdings direkt in der gleichen StraBe als Live-In-Pflegerin und Haushälterin bei Bekannten der Tante unterbringt, ,,blieb der Plan ein Plan (...) und außerdem ... plötzlich war es ja nicht mehr die Tante, um die ich mich kümmern musste, sondern dieses Ehepaar, die mich dafür bezahlt haben, sehr gut bezahlt haben!" Durch ihre anscheinend zufriedenstellende Arbeit im Haushalt des Ehepaars und darauf aufbauenden zusätzlichen Jobs als Putzhilfe, später auch als Kindermädchen und Live-Out-Haushälterin - also einem wachsenden Kundenkreis - kommt Maria die Idee, gemeinsam mit ihrer Cousine einen „Haushaltsservice“ anzubieten. Denn neben potentiellen Kunden „hatte ich auch diesen großen Bekanntenkreis, halt die Polen vom ZOB [Zentraler Omnibus Bahnhof Hamburg], die Leute, die man dort oder während der Busfahrten [zwischen Hamburg und Polen] trifft und die alle arbeiten wollen, mehr arbeiten wollen oder flexibler usw. Wir hatten dann diesen Service, der bestand aus einer Handynummer, aus Anzeigen in der Umsonstzeitung und sehr viel, na ja, Weiterreichen. D.h. jeder [der Haushalte] wusste immer noch gleich Bekannte, die ne Putzfrau suchen und so waren wir gut im Geschäft, besonders nach der Visumserleichterung [2001]! Die Busse aus Polen, da kommen ja manchmal mehrere pro Stunde an und wenn wir zu viel Arbeit hatten, dann bin ich da hin gegangen und habe Frauen unsere Arbeit angeboten, zur Not auch Frauen aus der Ukraine oder so, das ging dann halt irgendwie auf Russisch.“ Wie sich auf Nachfragen herausstellt, hat Maria diese Frauen 
allerdings nicht direkt an die Abeitgeber_innen weitergeleitet, sondern ist selbst als Vermittlerin aufgetreten, ,für ein, also für eine Provision ... also erst war alles provisorisch, dann für Provision!“ (Lacht und freut sich offensichtlich nicht nur über die Provision im finanziellen Sinne, sondern auch über das deutsche Wortspiel).

Mittlerweile ist Maria mit einem aus Polen stammenden Spätaussiedler verheiratet, hat zwei kleine Kinder und lebt dauerhaft und „legal“ in Hamburg. Allerdings vermittelt sie weiterhin als „Selbstständige“ irreguläre Putz-, Pflege- und Haushalthilfen und versucht sich dabei in „Expansion: neulich habe ich eine [Polin] nach Oslo vermittelt. Das lässt sich sicher ausbauen, solange wir [polnische Staatsbürger_innen] da noch nicht arbeiten dürfen. (...) Und ich muss mich auch kümmern, da die Frauen aus Rumänien jetzt hier [in Hamburg] den Markt kaputt machen... “.

Die Kategorie Geschlecht scheint hier in Kombination mit dem jungen Alter und dem gerade abgeschlossenen Studium, d.h. der Suche nach etwas „Neuem“, ausschlaggebend für Marias Übernahme der Pflege der Tante in Deutschland zu sein. Dies wird u.a. dadurch deutlich, dass ihre Vertretung bei der Tante durch ihre Cousine, nicht durch einen männlichen Verwandten, übernommen wird; zugleich weist dies auch auf eine innerfamiliäre „Sorgekette“ hin. ${ }^{6}$ Da sie anfänglich mit einem Touristenvisum regulär einreist und eine Familienangehörige unentgeltlich pflegt, wird die Kategorie Staatsbürgerschaft erst mit der Visumserleichterung bzw. dem EU-Beitritt Polens 2004 relevant bzw. „ökonomisch interessant", zumal Maria da bereits als irreguläre, aber bezahlte Haushaltshilfe etabliert ist. D.h. ab 2004 beginnt Maria, die Grenz- und Arbeitsmarktregulationen mithilfe der Kategorie Staatsbürgerschaft gezielt auszunutzen, um weitere Frauen aus Polen irregulär in deutsche Haushalte zu vermitteln. Dabei bleibt neben der Kategorie Staatsbürgerschaft das Frausein überaus relevant, wie ihre Suche nach „Frauen aus der Ukraine" am ZOB (nicht etwa nach polnischen Männern) deutlich macht.

Alina kam im Herbst 2006 mit einem Studienstipendium und -visum aus Weißrussland und hat die Zeit des Studiums „genutzt, um als Kindermädchen zu arbeiten. Immer, tagsüber, abends, nachts... manchmal sogar übers Wochenende oder wochenlang ohne Pausen." Dennoch schafft sie ihren Abschluss (Chemieingenieurswesen) - ist direkt danach gleichwohl ratlos: „Irgendwie stand für mich während

\footnotetext{
${ }^{6}$ Die transnationale Migration von Frauen, die als Pflege- oder Haushaltshilfen zwischen Ländern mit großen sozioökonomischen Unterschieden pendeln, wird auch als ,,(Global) Care Chain“ untersucht. Der Begriff verweist zumeist auf das Phänomen, dass Migrantinnen ihre eigenen Kinder oder pflegebedürftige Verwandte zuhause von Migrantinnen aus noch ärmeren Ländern versorgen lassen (vgl. Ehrenreich und Hochschild, 2002; Hochschild, 2000; Kofman und Raghuram, 2006). Dies ist bei keiner der hier Interviewten der Fall - gleichwohl liefert Marias Migrationsbiographie ein Beispiel für eine familiär organisierte und geschlechtlich codierte Care Chain.
}

des Studiums der Aufenthalt hier stärker im Vordergrund als die Uni, das wurde klar, als ich fertig war und das Visum ablief. Da bleiben war eigentlich ganz einfach, ich hab alles so gelassen, wie es ist. Mein Mietvertrag [im Studierendenwohnheim] lief sowieso schon länger über einen anderen Namen, Jobs habe ich noch mehr angenommen ... und so bin ich da reingerutscht." Mit Letzterem meint sie ihren illegalisierten Aufenthaltsstatus als so genannter Overstayer ${ }^{7}$, um den sie natürlich weiß, der ihr aber weniger im Alltag, als im Hinblick auf ihre Zukunft Sorgen macht: „Ohne Aufenthaltsrecht kann ich mich nicht von hier für Stellen hier [gemeint ist in beiden Fällen Deutschland] bewerben ... und ich kann momentan auch nicht nach Hause [Weißrussland] zurück, vielleicht kann ich dann nie wieder einreisen."

Während eines zweiten Interviews (18 Monate nach dem ersten) lebt Alina seit insgesamt 26 Monaten irregulär in Hamburg, wohnt und arbeitet seit fast einem Jahr als „Mädchen-für-Alles“ in einem Familienhaushalt am Stadtrand. „Dazu gehört auch die Pflege von Oma, deswegen verdiene ich da so viel. Aber das ist ideal, ich arbeite da sehr viel, ich verdiene da sehr viel ... und weil ich so viel arbeite, bin ich abends viel zu müde, um auszugehen, um Geld auszugeben. Mein gespartes Geld ist groß ... und damit kann ich hoffentlich nochmal was Richtiges lernen oder studieren, ganz offiziell?" Die Hoffnung auf „das Offizielle“, d.h. das Verlassen der Illegalität begründet Alina u.a. damit, dass sie ,,anerkannt“ ist: ,In der Straße meiner Familie, im Rewe, der Arzt von Oma und alle hier, alle wissen, dass ich nicht arbeiten darf, dass ich gar nicht da sein darf, aber alle wissen, dass es ohne uns nicht funktioniert oder nur sehr teuer funktioniert." 8

Die Erlangung einer universitären Qualifikation stellte für Alina ursprünglich den Anlass wie auch die rechtliche Grundlage für ihre Migration nach Deutschland dar, obgleich sie von Anfang an auch als Kindermädchen arbeitet und damit die an Kategorie Geschlecht gebundenen Assoziationen ausnutzt. Nach Studienabschluss erweist sich dann auch

\footnotetext{
${ }^{7}$ Die Typisierung als „Overstayer“ bezieht sich auf Migrant_innen, die nach Ablauf ihres Visums ohne Aufenthaltstitel im Zielland verbleiben. Ein anscheinend ebenfalls zunehmendes Phänomen ist die Einreise mit einem von Anfang an irregulären Visum: So erzählt Jelena, eine Richterin aus der Ukraine, die in unregelmäBigen Abständen zum Arbeiten als - wie sie sich selber bezeichnet - „Hausfrau“ nach Deutschland pendelt, dass sie immer mit Visa vom Schwarzmarkt nach Polen einreist und von dort „ohne Grenzkontrollen weiter nach Berlin oder direkt nach Hannover" fährt.

${ }^{8}$ Das ,öffentliche Geheimnis“ (Metz-Göckel, 2010a:11) der Beschäftigung illegalisierter Migrantinnen als Pflegekräfte bzw. das Wissen um das Funktionieren der häuslichen Pflege in Deutschland auf Grundlage irregulärer Beschäftigungen illustriert Karakayali (2010:16) sehr anschaulich: „Ein Pflegedienstleiter erklärte mir, dass er bereits mehrfach Telefonnummern irregulärer Migrantinnen an Angehörige Pflegebedürftiger weitergegeben hätte. Eine der irritierendsten Begegnungen war die mit einer Polizeibeamtin, die mir Kontakte zu irregulär arbeitenden Haushaltsarbeiterinnen vermittelte.“
} 
Letztere als besonders wertvoll und die hohe Bedeutung des Frauseins steigt im Zusammenwirken mit ihrem Illegalisiertsein als Weißrussin nach Ablauf des Visums, wohingegen ihr Universitätsabschluss - zumindest zeitweise - irrelevant geworden ist.

Cornelia hat 2006 eine Ausbildung zur Krankenschwester in Sibiu (Hermannsburg; Rumänien) abgeschlossen und dort auch kurzzeitig im Krankenhaus gearbeitet. „Aber es war schrecklich, es gab nichts, keine richtigen Medikamente, keine Zeit für die Kranken ..., auch kein Geld für uns [die Pfleger_innen].“ Da ihr Großvater der deutschsprachigen Minderheit in Rumänien angehört und als Kind deutsch mit ihr gesprochen hat, wollte sie eigentlich Deutsch-Lehrerin werden. „Statt dem Studium hab ich dann aber erstmal das im Krankenhaus gemacht, weil ich dachte, damit kann ich schnell Geld verdienen und erstmal zuhause bleiben'. Geld verdienen ... das ging nicht, zuhause bleiben dann auch nicht ... und dann bin ich nach dem [EU-] Beitritt ziemlich schnell nach Deutschland, weil ich ja deutsch sprechen kann. Hier ging das schnell, die Rumänen helfen sich, helfen sich gegenseitig ... und in meinem Haus [-halt; Unterstützung und Pflege eines älteren Ehepaares in einer norddeutschen Kleinstadt] freuen die sich über mein lustiges Deutsch." Cornelia lebt und arbeitet seit fast vier Jahren in diesem Haushalt, pendelt davon die letzten drei regelmäßig alle sechs Wochen für sieben bis zehn Tage nach Sibiu und hofft bzw. wartet, dass sie so die Zeit bis Anfang 2014 überbrücken kann. Denn dann will sie „Arzt oder Lehrer studieren“ ... und wenn das nicht geht ,mit meinem Beruf die deutsche Ausbildung in Altenpflege machen".

Dieses Abwarten auf die formale Legalisierung durch die Gleichbehandlung der EU-Beitrittsländer von 2007 zum 1. Januar 2014, in diesem Fall also Rumänien, hat Sonya, eine Interviewpartnerin aus Polen, die zehn Jahre irregulär als Live-In-Pflegerin für ein älteres Ehepaar in Deutschland tätig war und deren Aufenthaltsstatus sich 2004 legalisierte, folgendermaßen beschrieben: „Erst habe ich die Grenze unerlaubt überschritten und jetzt hat mich die Grenze übertreten, indem sie weggefallen ist.“

Zusammenfassend lässt sich für alle der interviewten Migrantinnen feststellen, dass die Kategorie Geschlecht in Interdependenz mit der Kategorie Staatsbürgerschaft ausschlaggebend für den Einstieg in die irreguläre Haushaltsarbeit war - und dass das , junge Alter" im Sinne von Ungebundenheit, Offenheit und Neugierigkeit die Erlangung bzw. das Einsetzen einer beruflichen oder universitären Qualifikation in den Hintergrund treten lässt. Während für Maria ihre ursprüngliche Qualifikation spätestens mit Etablierung ihrer Vermittlerinnenrolle für Haushaltshilfen bedeutungslos geworden ist, war die Erlangung eines Abschlusses in Deutschland für Alina erst Anlass für den ersten Grenzübertritt und stellt für Cornelia in Kombination mit ihren Deutschkenntnissen die Grundlage sowohl für ihre derzeitige Arbeit als irreguläre Pflegekraft als auch für die Hoffnung auf eine zukünftige Professionalisierung in Deutschland dar.
Für alle drei hier vorgestellten Care-Migrantinnen wiederum spielt die Bereitschaft zur professionalisierten Domestizierung bzw. zum Einsatz professionalisierter Emotionen und zur ,emotional labour“ (Dyer et al., 2008) eine entscheidende Rolle.

Die illustrierend skizzierten Beispiele von Migrationsbiographien bestätigen die Tendenz, dass Care-Tätigkeiten speziell von jungen Osteuropäerinnen genutzt werden, um irregulär oder illegalisiert - Erfahrungen zu sammeln und „Career mit Care“ als Haushaltshilfe oder Pflegekraft zu machen (ausführlicher, siehe Strüver, 2011). Die transnationale Pendelmigration als Haushaltshilfen kann dabei als Strategie von jungen Osteuropäerinnen eingeordnet werden, die durch sozioökonomische Disparitäten zwischen Herkunftsund Zielland - sowie durch die zwischen ihnen etablierten Grenzregime und daran gekoppelte Aufenthalts- und Arbeitsbestimmungen reguliert wird. Von besonderem Interesse sind dabei die sich beim Grenzübertritt ändernden Bedeutungen und Bewertungen der sozialstrukturellen Kategorien Geschlecht, Alter und Staatsbürgerschaft bzw. die sich verändernden Konstellationen innerhalb einer Person.

\section{Transnationale Pendelmigration und Subjektivierungsprozesse}

Transnationalisierung bedarf expliziter Raumbezüge und nationalgesellschaftlicher Kontextualisierungen, die u.a. in sozioökonomischen Unterschieden und Arbeitsmarktregulierungen zwischen Staaten zu finden sind (s.o.). Die Pendelmigration osteuropäischer Haushaltshilfen als individualisierte Strategie innerhalb transnationaler Netzwerke stellt die Annahme vom nationalstaatlichen - bzw. EU-homogenen Containerraum als rahmendes Moment ökonomischer und politischer Organisation in Frage. Der sogenannte ,,methodologische Kosmopolitismus" thematisiert das weiterhin wirkmächtige Prinzip nationaler Grenzregime und die daran gebundene (Re-)Produktion geopolitischer und -ökonomischer Ungleichheiten (vgl. Glick Schiller und Wimmer, 2003).

Für die beruflich qualifizierten, sich dann aber als Haushaltshilfen umorientierenden jungen Migrantinnen aus Osteuropa basiert ihr Handlungsspielraum u.a. auf dem Wechsel von beruflicher Professionalisierung zu Domestizierung, z.T. in Form von ,professionalisierter Domestizierung“ oder mithilfe „professionalisierter Emotionen“. Die strategische Migration von Haushaltsarbeiterinnen beruht somit einerseits auf nationalstaatlichen Grenzen und Unterschieden, um überhaupt entstehen zu können; sie bedarf andererseits aber auch einer Forschungsperspektive, die Europa als ,supranational stabilisierten Ungleichheitsraum“ thematisiert (vgl. Beck und Poferl, 2010; Mau und Verwiebe, 2010). Eine transnationale Perspektive in der aktuellen Migrationsforschung erfordert damit die explizite Thematisierung von Prozessen der Subjektkonstitution - als Prozesse, in denen sich Ungleichheitskategorien wie Staatsbürgerschaft, Geschlecht, 
Alter und Status/Klasse durchkreuzen. Denn die Subjektpositionen von osteuropäischen Pendelmigrantinnen sind u.a. bestimmt durch Raumrelationen: sie variieren mit jedem Grenzübertritt in ihren Konstellationen. D.h. die Migration wird durch die Unterschiede zwischen Herkunfts- und Arbeitsland, durch den anhaltenden räumlichen wie auch sozialen Grenzübertritt initiiert.

\subsection{Geschlechtlich codierte Migration}

Grenzüberschreitende Arbeitsmigration und Wirtschaftsbeziehungen sind auf allen Maßstabsebenen durch geschlechtliche Codierungen strukturiert, so dass sich durch zunehmende Globalisierung und Europäisierung, aber auch durch ökonomische Krisen Subjektivitäten und Geschlechternormen neu konfigurieren (vgl. Wichterich, 2010). Dazu gehören einerseits die stark gewachsene sozioökonomische Integration von Frauen, andererseits aber auch die veränderten Geschlechterarrangements und -rollen, in der politischen Öffentlichkeit wie auch im Privatbereich. Gerade in Letzterem haben die verbesserten Qualifikations- und Berufsmöglichkeiten von Frauen zu einem steigenden Bedarf an familienähnlichen entlohnten Fürsorgetätigkeiten in Deutschland geführt, der im Zusammenspiel mit dem oben angedeuteten Rückbau des deutschen Wohlfahrtsstaates sowie dem demographischen Wandel durch größtenteils weibliche migrantische Haushaltshilfen abgedeckt wird. ${ }^{9}$ Dieses Phänomen wird teilweise als „Feminisierung der Migration“ tituliert, das sich gleichermaßen auf die Quantität (steigende Zahl migrierender Frauen) wie die „Qualität“ (vorrangig flexible, prekäre Beschäftigungsverhältnisse) bezieht (vgl. Han, 2003; konkret im Hinblick auf migrantische Haushaltshilfen, siehe auch Ehrenreich und Hochschild, 2002; Kofman und Raghuram, 2006; Lutz, 2008; Pessar und Mahler, 2003; PfauEffinger und Rostgaard, 2011; Sassen, 2000). Neben der analytisch wichtigen Betonung der unterschiedlichen Migrationsgründe und -muster von Männern und Frauen sowie der Hinterfragung der Konnotation von weiblicher Migration mit Haushaltsarbeit beinhaltet diese Zuschreibung der „Feminisierung“ bzw. die Titulierung als „geschlechtsspezifische“ Migration gleichwohl eine Essentialisierung der Kategorie Geschlecht. Damit einher geht die Reifizierung der Geschlechterdifferenz, die darin besteht, dass Geschlecht zwar als sozial hervorgebrachte, aber dennoch unabhängige Kategorie verstanden wird, mittels derer bestimmte Verhaltensweisen erklärt - oder sogar naturalisiert - werden. ${ }^{10}$ Die hier vorgebrachte Kritik an diesem Ansatz verweist wiederum auf anti-essentialistische diskurs- und performativitäts-

\footnotetext{
${ }^{9}$ Einschließlich des Phänomens der „Care Chains“ (s.o.)

${ }^{10}$ Naturalisierung beinhaltet hier die Annahme, dass Frauen qua Geschlecht eine „naturgegebene“ Disposition für Haushaltsund Sorgearbeit hätten. Die momentan beobachtbaren Formen von transnationaler Arbeitsmigration von Osteuropäerinnen nach Deutschland scheinen diese Annahme zu bestätigen - doch ist diese Bestätigung nicht als „Naturzustand“ zu lesen.
}

theoretische Subjektkonzeptionen, die Subjekte und Identitäten als Effekte diskursiver Aushandlungsprozesse und performativer Sprechakte („Anrufungsprozesse“) konzipieren. Dementsprechend thematisiert dieser Beitrag weniger „geschlechtsspezifische Geographien der Migration“ (zusammenfassend, siehe Hillmann und Wastl-Walter, 2011), als geschlechtlich codierte Muster der Arbeitsmigration im Kontext der sozioökonomischen Transformationen in Europa sowie am Beispiel von Subjektkonstitutionen in der Krise.

\subsection{Situierte Subjektpositionierungen}

Im Prozess der transnationalen Migration verbinden sich vertikale und horizontale Ungleichheitskategorien: die „Ressourcenausstattung" einer Person mit dem Bedarf an dieser Ressource in bestimmten nationalräumlichen Kontexten (z.B. gering entlohnte Unqualifizierte oder Illegalisierte sowie auch entlang von Geschlechts- oder Alterskategorien Stereotypisierte). Dabei geht es nicht um die Addition oder den Vergleich von Ungleichheiten auf unterschiedlichen Maßstabsebenen, sondern darum, dass sozioökonomische Ungleichheiten zwischen Nationalstaaten sowie zwischen Identitätskategorien (wie Geschlecht, Alter oder Staatsbürgerschaft) multiskalar wirkmächtig sind.

Bevor dies in Abschnitt 5 erläutert wird, bedarf es eines kurzen theoretischen Exkurses in die Prozesse der Subjektkonstitution als Ausdruck des Wechselverhältnisses von Herrschafts- und Selbsttechniken: In Foucaults Analysen von Machtverhältnissen werden Subjekte durch diskursivvermittelte gesellschaftliche Normen entlang der Prinzipien von Ein- und Ausschluss konstituiert, einschließlich der Bedeutungen ,individueller“ Erfahrungen. Diskurse verweisen dabei auf das Prinzip von Sprache als Repräsentationssystem, durch das gesellschaftliche Ordnungen und ihre sozialen und politischen Konsequenzen sowie verkörperte Subjektidentitäten konstruiert werden (vgl. Foucault, 1977). In der Weiterentwicklung der Machtdefinition durch die Konzeption von Gouvernementalität als Regierungskunst geht es Foucault dann verstärkt um Selbsttechnologien als Teil von Subjektivierungspraktiken, ,mit denen Menschen nicht nur die Regeln ihres Verhaltens festlegen, sondern sich selber zu transformieren, sich in ihrem besonderen Sein zu modifizieren und aus ihrem Leben ein Werk zu machen suchen" (Foucault, 1986:18). Selbsttechnologien sind eingebettet in gesellschaftliche Herrschaftsverhältnisse - bilden jedoch ein Gegengewicht dazu, sie setzen der Fremdbeherrschung die Selbstermächtigung entgegen. Das Zusammenwirken von Fremd- und Selbsttechnologien wiederum beschreibt Foucault mit dem Begriff der „Regierung“ im Sinne von Führung: Der Begriff verbindet Macht und Subjektivität, Herrschafts- und Selbsttechniken. Das derart „regierte“ Subjekt im Neoliberalismus ist ein „Unternehmer seiner selbst“ (Foucault, 2004:314) und verweist auf die Frage ,,welches die Formen und die Modalitäten des Verhältnisses zu sich 
sind, durch die sich das Individuum als Subjekt konstituiert und erkennt" (Foucault, 1986:29).

In Erweiterung der diskurstheoretischen Konzeption von Sprache als Bezeichnungs- und Bedeutungspraxis konzentriert sich Butler $(1991,1997)$ stärker auf den performativen Charakter von Sprache, auf die Produktion sozialer und räumlicher Wirklichkeiten im „Vollzug“ Ihr Sinn geht ihnen nicht voraus, sondern entsteht im performativen (Sprech-)Akt. Eine Äußerung beinhaltet demnach stets die Ausführung des Gesprochenen, zugleich sind es alltägliche, routinisierte, oft auch ritualisierte Auffïhrungen, mittels derer diskursive Normen fortgeschrieben werden. Performativität ist in diesem Sinne die diskursive Praxis, die die körperlich-materiellen Identitäten eines Subjekts „real“ werden lässt. Die Subjektivitäts- und Identitätsbildung befindet sich in einem ständigen Prozess von Bestätigung oder Neudefinition des Selbst, und dieser Prozess wird durch ökonomische, politische, soziale und kulturelle Diskurse geprägt. Subjektidentitäten im Sinne Butlers sind daher nicht kohärent, sondern kontingent; sie stellen keine fixe Einheit dar, sondern basieren auf einer Vielzahl von Relationen und Positionierungen. „Die Theorien der feministischen Identität, die eine Reihe Prädikate wie Farbe, Sexualität, Ethnie, Klasse und Gesundheit ausarbeiten, setzen stets ein verlegenes usw. an das Ende ihrer Liste. Durch die horizontale Aufzählung der Adjektive bemühen sich diese Positionen, ein situiertes Subjekt zu umfassen; doch gelingt es ihnen niemals, vollständig zu sein. Dieses Scheitern ist aber äußerst lehrreich, denn es stellt sich die Frage, welcher politische Impetus aus dem usw. abzuleiten ist, das so oft am Ende dieser Zeilen auftaucht. Tatsächlich ist es ebenso ein Zeichen der Erschöpfung wie ein Zeichen für den unbegrenzbaren Bezeichnungsprozess selbst.“ (Butler, 1991:210, Hervorh. A.S.)

\section{Intersektionale Subjektkonstitution}

Diese Vielzahl von Identitätsbezeichnungen verweist neben der Relationalität auch auf die Komplexität und Heterogenität von Subjektpositionen, in denen sich Kategorien wie Geschlecht mit anderen Identitäts- und Differenzzuschreibungen wie Alter und Staatsangehörigkeit durchkreuzen. Diese Durchkreuzung wird mit dem Konzept der Intersektionalität zu erfassen versucht: Soziale Kategorien werden als hierarchisierende Unterscheidungsmerkmale und interdependente Herrschaftsverhältnisse verstanden, die sich gegenseitig verstärken oder abschwächen können und prozessual in einer offenen Matrix miteinander verbunden sind. Diese Matrix umfasst sowohl institutionalisierte wie auch informalisierte und alltägliche Ein- und Ausschlussmechanismen auf unterschiedlichsten sozialen wie räumlichen Maßstabsebenen - sie besteht aus den Wechselwirkungen zwischen gesellschaftlichen Strukturen, diskursiven Normen und individualisierten Praktiken und ist immer kontextspezifisch (vgl. Walgenbach et al., 2012; Winker und Degele, 2009).
Für das hier vorgestellte empirische Beispiel umfasst sie die europäischen Arbeitsmarkt-, Grenz- und Migrationsregime einerseits sowie die individuelle Bereitschaft junger qualifizierter Osteuropäerinnen zur räumlichen wie sozialen Mobilität andererseits. Dabei ermöglicht die Einbeziehung von Staatsbürgerschaft als Strukturkategorie eine zusätzliche Perspektive, da die sozioökonomische Position über diese Kategorie - sowie die sich daraus ggf. ableitende Illegalität - bei den osteuropäischen Haushalthilfen in Deutschland zwischen Herkunfts- und Arbeitsland variiert bzw. durch diesen Unterschied im Zusammenwirken mit „Frau-Sein“ und „Jung-Sein“ die soziale wie räumliche Mobilität initiiert. Das Intersektionalitäts-Konzept wird daher auch verwendet, um einer Homogenisierung der Kategorie Geschlecht bzw. Frau zuvorzukommen und um ,geschlechtsspezifische“ Annahmen nicht zu reifizeren, sondern zu de-essentialisieren und zu pluralisieren. Zudem ermöglicht die Einbeziehung räumlicher Relationen in der Erforschung sozioökonomischer Ungleichheiten einerseits und interdependenter Identitätskategorien andererseits die Erfassung der sich beim Grenzübertritt verändernden Konstellationen intersektionaler Subjektpositionen von Migrantinnen. Dabei ist die Dimension des Räumlichen auf den Ebenen der gesellschaftlichen „Regierung“ wie der individualisierten „Führung“ so eng mit der Identitätskonstitution verwoben, dass die Interdependenzen von Kategorien wie Klasse, Geschlecht und Alter um die des Raumes erweitert werden sollte - ,intersectionality works out in different ways in different places" (McDowell, 2008:504; siehe auch Richter, 2011; Valentine, 2007). Knapp (2010:240) fordert dementsprechend einen ,spatial turn (der) die feministische Intersektionalitätsdiskussion bereichern" würde und verweist auf die räumlichen Konstruktionen und Dimensionen sozialer Differenz- und Machtverhältnisse, z.B. zwischen Nationalstaaten und - wie hier ausgeführt - innerhalb transnationaler Migrationsregime.

Für die Durchführung intersektionaler Analysen stellt sich meist die Frage, auf welcher Ebene (Strukturen, Diskurse oder Individuen) anzusetzen sei. In Anlehnung an McDowell (2008), Valentine (2007) und Winker und Degele (2009) wurde im vorliegenden Fallbeispiel am einzelnen Subjekt angesetzt. Dies geschah mit dem Ziel zu erfassen, welche Identitätskategorien in welchen sozialen, ökonomischen und räumlichen Kontexten wie bedeutsam werden bzw. in verschiedenen Alltagssituationen genutzt werden. „Such an analysis means asking questions about what identities are being ,done', and when and by whom [and where], evaluating how particular identities are weighted or given importance by individuals at particular moments and in specific [social and spatial] contexts“ (Valentine, 2007:15; Ergänzungen A.S.).

Die Konzentration auf die anhaltende Interaktion unterschiedlicher Kategorisierungen im Prozess der Subjektkonstitution zielt dabei nicht auf einen methodologischen Individualismus, sondern auf die Verbindung von Mikro- mit Makroanalysen in ,intersektionalen Mehrebenenanalysen“ (Winker und Degele, 2009), die die Wechselwirkungen 
ungleichheitsgenerierender Gesellschaftsstrukturen und Identitätskategorien rekonstruieren, so dass Differenz- und Identitätskategorien ,als interdependent“ (Walgenbach et al., 2012) begriffen werden. Vor diesem Hintergrund betont bspw. Marina Richter (2011) die Notwendigkeit intersektionaler Analysen in der Migrationsforschung, um zum einen die Komplexität von „Topographien der Ungleichheit" zu erfassen und zum anderen Kategorisierungen von „Klasse“ bzw. Berufs- und Bildungsstatus auch jenseits formaler Berufs- oder Universitätsabschlüsse anzuerkennen. ${ }^{11}$ Münst (2008) sieht schließlich in der Kombination von transnationalen und intersektionalen Ansätzen in der Migrationsforschung das Potenzial für die Erforschung dieser „Topographien von Ungleichheiten“, die Erforschung sich verändernder Identitätspositionen zwischen Herkunftsund Zielland. Diese Identitäts- bzw. „Feldpositionen verbinden sich im Migrationsprozess - in der Person der Migrantin/des Migranten - zu einer dritten Position, in der sich die Prozesse und Logiken der Migration, die kontextund ortsabhängigen Dynamiken zwischen Exklusion und Inklusion manifestieren und insofern auch erschließen lassen" (Münst, 2008:46; siehe auch McDowell, 2008, Nagar et al., 2002 sowie Riaño, 2011).

\section{6 „Die Grenze übertreten“: Europäische Grenz- und Migrationsregime}

Dieser Beitrag hat den individuellen Umgang von migrantischen Haushaltsarbeiterinnen mit den europäischen Grenz, Migrations- und Arbeitsmarktregimen fokussiert, um die gesellschaftliche Konstitution von Subjektivierungsprozessen konzeptionell sowie den Umgang mit Krisensituationen als Folgen der geopolitischen und -ökonomischen Ungleichheitsverhältnisse innerhalb Europas zu verstehen.

Die irreguläre Haushalts- und Pflegearbeit von Osteuropäerinnen in Deutschland macht deutlich, dass die europäische Aufenthalts- und Arbeitsmarktpolitik nicht in dem Maße restriktiv und exkludierend wirkt, wie sie formuliert ist. Vielmehr führt sie zur Entstehung neuer europäischer Grenz- und Migrationsregime, die eine Temporalisierung, Flexibilisierung und teilweise Illegalisierung von Arbeitsmigrant_innen produzieren - und sich zugleich durch diese Aufenthalte stabilisieren. So wird von einer „Komplizenschaft" (Lutz und Palenga-Möllenbeck, 2010) des deutschen Staates gesprochen, da die Tätigkeiten der Migrantinnen die Dienstleistungslücken im Bereich von Haushalt, Betreuung und Pflege „im Verborgenen“ schließen, ohne dass dafür später Ansprüche auf Arbeitslosen- oder Rentenzahlungen erhoben werden können. Die systematische Illegalisierung von Migrant_innen durch das europäische Grenzregime re-

\footnotetext{
${ }^{11} \mathrm{Im}$ vorliegenden Beispiel wären das u.a. die genannten professionalisierten Emotionen, aber auch die durch das anhaltende Pendeln stetig wachsenden Kontakte zu anderen Migrant_innen bzw. irregulären Haushaltsarbeiterinnen.
}

sultiert somit in einer rechtlichen und politischen Exklusion bei gleichzeitiger ökonomischer „Inklusion“ in den irregulären, prekären Arbeitsmarkt für Haushalts- und Pflegekräfte. Oftmals unberücksichtigt bleibt dabei allerdings die subjektive Dimension von grenzüberschreitender Mobilität, z.B. die selbstorganisierte Pendelmobilität der transnationalen Haushaltshilfen im Rotationsprinzip. Diese Selbstorganisation verweist dabei weniger auf Subjekte als autonom Handelnde als auf eine Form der Selbst-Organisation, die neben dem Organisieren des Pendelns vor allem die gesellschaftliche Konstitution von Subjektivitäten und ihren Positionen innerhalb gesellschaftlicher Machtstrukturen impliziert. „In diesem Sinne sollte auch eine Analyse von Grenzregimen aus der Perspektive der Migration, und das heißt aus der Perspektive grenzüberschreitender Biografien und Aktivitäten konzipiert sein. Nur so wird es möglich, Praktiken der Migration (und damit gemeint sind Diskurse, Machtverhältnisse und Politikformen und nicht nur die ,empirischen ' Praktiken der MigrantInnen) in eine Theorie der, Autonomie der Migration" einzubeziehen" (Tsianos und Hess, 2010:244; vgl. auch Hess, 2009; Karakayali, 2008). „Autonomie" verweist demnach nicht auf eine Unabhängigkeit von gesellschaftlichen und zwischenstaatlichen Machtverhältnissen, sondern auf den Einfluss der Migrationsbewegungen auf Grenzregime. Eine Grenzregime-Analyse wiederum macht eine - sozial wie räumlich - multiskalare und intersektionale Perspektive auf die Subjektivierungsprozesse von Migrant_innen möglich und nötig, einschließlich der die Grenze und die transnationale Migration konstituierenden sozioökonomischen Unterschiede.

Grenz- und Migrationsregime erweisen sich als verdichtete Effekte von Machtverhältnissen zwischen verschiedenen Institutionen, sozialen Akteuren und deren Praktiken. Dabei besteht die Produktivität eines Grenzregimes in der Regulation grenzüberschreitender Arbeitsmobilität, die, mit den Worten Foucaults, darin besteht, ,die Zirkulationen zuzulassen, zu gewährleisten, sicherzustellen“" (Foucault, 2004, zitiert in Tsianos und Hess, 2010:247f), auch im Sinne einer „,institutionalisierten Durchlässigkeit der Grenze“ (Karakayali und Tsianos, 2005:50; siehe auch Transit Migration Forschungsgruppe, 2007). D.h. die Wirkungsweise des europäischen Grenzregimes besteht nicht in der konsequenten Kontrolle und Schließung der Grenzen für „Außenseiter“. Neben den hier nicht berücksichtigten „Ausnahmeregelungen“ (für spezielle Branchen oder Herkunftsregionen) scheint bspw. die deutsche Grenz- und Migrationspolitik auf eine ,gezielte Illegalisierung" von osteuropäischen Haushaltshilfen bzw. auf eine „Institutionalisierung der informalisierten Durchlässigkeit der Grenzen“" ausgerichtet zu sein.

Die „transnationale Wende“ in der Migrationsforschung beinhaltet neben der Infragestellung des methodologischen Nationalismus eine verstärkte Konzentration auf den Subjektstatus von Migrant_innen bzw. auf transnational agierende Subjekte. Allerdings bleiben in der Fokussierung auf transnationale soziale Räume und transmigrantische 
Netzwerke oftmals die Bedeutungen nationalstaatlicher Grenzen bzw. der Einfluss des Überschreitens dieser Grenzen auf den anhaltenden Prozess der Identitätskonstellation und Subjektkonstitution unberücksichtigt. Ein Zusammendenken globaler und lokaler sozioökonomischer Transformationen sowie (trans-)nationaler Arbeitsmarkt-,,Regulationen“ erweitert diesen Ansatz im Hinblick auf räumlich und sozial verschiedene, vor allem aber sich verschiebende Formen von Identitätskonstellationen. Pessar und Mahler (2003:815) plädieren in Anlehnung an Massey's „Power Geometries of Mobility“ (1994) für eine Analyse von geschlechtlich codierten transnationalen Migrationsprozessen unter expliziter Einbeziehung der Rolle von Staatsbürgerschaft: Gendered Geographies of Power ,capture our understanding that gender operates simultaneously on multiple spatial and social scales (e.g. the body, the family, the state). It is both within the context of particular scales as well as between and among them that gender ideologies and relations are reaffirmed, reconfigured or both."

Vor diesem Hintergrund ist die transnationale Pendelmobilität von Osteuropäerinnen weniger zu verstehen als ,gezielte Subversionsstrategie“, denn (1) als Reaktion auf die ungenügenden Beschäftigungsmöglichkeiten im Herkunftskontext, (2) als Alltagspraktik im Umgang mit der ,,institutionalisierten Durchlässigkeit" von Grenzen sowie (3) als Umgang mit den sich durch den Grenzübertritt verändernden Wertigkeiten der Kategorien Staatsbürgerschaft, Geschlecht, Alter und Berufsqualifikation, einschließlich nicht formalisierter Softskills wie professionalisierte Emotionen. Die Praktiken der andauernden Grenzüberschreitungen, die das Grenzregime zwangsläufig dynamisieren, ,lassen sich als Ausdruck eines pragmatischen Alltagskosmopolitismus verstehen, der auf der Grundlage sozialer Erfahrungen das Wissen und die Kompetenzen erwirbt, Grenzen - in ihrer geopolitischen wie in ihrer kulturell konstruierten Gestalt - taktisch und kreativ gegen sich selbst auszuspielen“ (Römhild, 2007:217). Dies verweist auch wie im Falle von Maria darauf zu spekulieren, dass die irreguläre Arbeit anfänglich zwar auf einem Provisorium basiert, dann aber in anhaltenden ökonomischen Gewinnen durch Provisionen resultiert oder auf ein Abwarten (wie von Cornelia oder Sonya), dass ,,mich die Grenze übertreten [hat], indem sie weggefallen ist".

Ein derartiger pragmatischer Alltagskosmopolitismus verweist schließlich auf eine „Gouvernementalisierung der Migrationspolitik“ (Transit Migration Forschungsgruppe, 2007) und bezieht sich auf das Foucaultsche Prinzip der „Regierung“ von Subjekten: auf das Zusammenwirken von Herrschafts- und Selbsttechniken im Prozess der Subjektkonstitution sowie auf die performative Produktion von Wirklichkeiten „im Vollzug“ - auf den unabgeschlossenen und „unbegrenzten“ Prozess der Subjektkonstitution.

Edited by: O. Söderström

Reviewed by: two anonymous referees

\section{Literatur}

Baas, T. und Brücker, H.: Arbeitnehmerfreizügigkeit zum 1. Mai 2011. Mehr Chancen als Risiken für Deutschland, Aktuelle Analysen aus dem Institut für Arbeitsmarkt- und Berufsforschung, Nürnberg, No. 10, 2011.

Bauder, H.: "Brain Abuse", or the devaluation of immigration labour in Canada, Antipode, 35, 699-717, 2003.

Beck, U. und Poferl, A. (Hrsg.): Große Armut, großer Reichtum. Zur Transnationalisierung sozialer Ungleichheit, Suhrkamp, Frankfurt/Main, 2010.

Butler, J.: Das Unbehagen der Geschlechter, Suhrkamp, Frankfurt/Main, 1991.

Butler, J.: Körper von Gewicht. Die diskursiven Grenzen des Geschlechts, Suhrkamp, Frankfurt/Main, 1997.

Dyer, S., McDowell, L., und Batnitzky, A.: Emotional labour/body work: the caring labours of migrants in the UK's National Health Service, Geoforum, 39, 2030-2038, 2008.

Ehrenreich, B. und Hochschild, A. (Hrsg.): Global Woman. Nannies, Maids, and Sex Workers in the New Economy, Granta, London, 2002.

Foucault, M.: Der Wille zum Wissen. Sexualität und Wahrheit I, Suhrkamp, Frankfurt/Main, 1977.

Foucault, M.: Der Gebrauch der Lüste. Sexualität und Wahrheit II, Suhrkamp, Frankfurt/Main, 1986.

Foucault, M.: Geschichte der Gouvernementalität, Bd. II. Die Geburt der Biopolitik, Suhrkamp, Frankfurt/Main, 2004.

Glick Schiller, N. und Wimmer, A.: Methodological nationalism, the social sciences and the study of migration, Int. Migr. Rev., 37, 576-610, 2003.

Han, P.: Frauen und Migration, utb, Stuttgart, 2003.

Hess, S.: Globalisierte Hausarbeit. Au-pair als Migrationsstrategie von Frauen aus Osteuropa, VS Verlag für Sozialwissenschaften, Wiesbaden, 2009.

Hillmann, F. und Wastl-Walter, D.: Geschlechtsspezifische Geographien der Migration, Berichte zur deutschen Landeskunde, 85, 5-23, 2011.

Hochschild, A.: Global care chains and emotional surplus value, in: On the Edge. Living with Global Capitalism, Herausgeber: Hutton, W. und Giddens, A., Jonathan Cape, London, 130-146, 2000.

Karakayali, J.: Transnational haushalten. Biographische Interviews mit care workers aus Osteuropa, transcript, Bielefeld, 2010.

Karakayali, S.: Gespenster der Migration. Zur Genealogie illegaler Einwanderung in der Bundesrepublik Deutschland, transcript, Bielefeld, 2008.

Karakayali, S. und Tsianos, V.: Mapping the Order of New Migration. Undokumentierte Arbeit und die Autonomie der Migration, Peripherie, 97/98, 35-64, 2005.

Knapp, G. A.: „Intersectional Invisibility“, in: Fokus Intersektionalität, Herausgeber: Lutz, H., Vivar, M., und Supik, L., VS Verlag für Sozialwissenschaften, Wiesbaden, 223-243, 2010.

Kofman, E. und Raghuram, P.: Gender and Global Labour Migrations: Incorporating Skilled Workers, Antipode, 38, 282-303, 2006.

Lutz, H.: Vom Weltmarkt in den Privathaushalt. Die neuen Dienstmädchen im Zeitalter der Globalisierung, Budrich, Opladen, 2008. 
Lutz, H. und Palenga-Möllenbeck, E.: Care Work Migration in Germany: Semi-Complinance and Complicity, Social Policy and Society, 9, 419-430, 2010.

Massey, D.: Space, Place and Gender, Polity, Cambridge, 1994.

Mau, S. und Verwiebe, R.: European Societies. Mapping Structure and Change, Policy Press, Bristol, 2010.

McDowell, L.: Thinking through work: complex inequalities, constructions of difference and trans-national migrants, Prog. Hum. Geogr., 32, 491-507, 2008.

Metz-Göckel, S.: Einleitung, in: Migration als Ressource. Zur Pendelmigration polnischer Frauen in Privathaushalte der Bundesrepublik, Herausgeber: Metz-Göckel, S., Kalwa, D., und Münst, S., Budrich, Opladen, 11-26, 2010a.

Metz-Göckel, S.: Arbeitspendeln und Lebensarbeit, in: Migration als Ressource. Zur Pendelmigration polnischer Frauen in Privathaushalte der Bundesrepublik, Herausgeber: Metz-Göckel, S., Kalwa, D., und Münst, S., Budrich, Opladen, 27-59, 2010b.

Münst, S.: Intersektionalität als Perspektive der Migrationsforschung, Femina Politica, 12, 41-54, 2008.

Nagar, R., Lawson, V., McDowell, L., und Hanson, S.: Locating Globalization. Feminist (Re)readings of the Subjects and Spaces of Globalization, Econ. Geogr., 78, 257-284, 2002.

Pessar, P. und Mahler, S.: Transnational Migration: Bringing Gender In, Int. Migr. Rev., 37, 812-846, 2003.

Pfau-Effinger, B. und Rostgaard, T.: Care between work and welfare in European societies, Palgrave, Basingstoke, 2011.

Pohl, C.: Der zukünftige Bedarf an Pflegearbeitskräften in Deutschland, Zeitschrift für Bevölkerungswissenschaft, 35, 357-378, 2010.

Rerrich, M.: Die ganze Welt zu Hause. Cosmobile Putzfrauen in privaten Haushalten, Hamburger Edition, Hamburg, 2006.

Riaño, Y.: Barrieren aufbrechen: Erfahrungen und Strategien von qualifizierten Migrantinnen beim Zugang zum Schweizer $\mathrm{Ar}$ beitsmarkt, Berichte zur deutschen Landeskunde 85, 25-48, 2011.
Richter, M.: Topographien der Ungleichheit: Dequalifikation als rein weibliches Phänomen?, Berichte zur deutschen Landeskunde, 85, 49-59, 2011.

Römhild, R.: Alte Träume, neue Praktiken. Migration und Kosmopolitismus an den Grenzen Europas, Turbulente Ränder. Neue Perspektiven auf Migration an den Grenzen Europas, Herausgeber: Transit Migration Forschungsgruppe, transcript, Bielefeld, 211-222, 2007.

Sassen, S.: Women's burden: Counter-geographies of globalization and the feminization of survival, J. Int. Aff., 53, 503-524, 2000.

Statistisches Bundesamt: Pflegestatistik 2007, Wiesbaden, 2009.

Strüver, A.: Zwischen Care und Career - Haushaltsnahe Dienstleistungen von transnationalen mobilen Migrantinnen als strategische Ressourcen, Zeitschrift für Wirtschaftsgeographie, 55, 193-206, 2011

Transit Migration Forschungsgruppe (Hrsg.): Turbulente Ränder. Neue Perspektiven auf Migration an den Grenzen Europas, transcript, Bielefeld, 2007.

Tsianos, V. und Hess, S.: Ethnographische Grenzregimeanalyse, in: Grenzregime. Diskurse, Praktiken, Institutionen in Europa, Herausgeber: Hess, S. und Kasparek, B., Assoziation A, Berlin, 243264, 2010.

Valentine, G.: Theorizing and researching intersectionality: A challenge for feminist geography, Prof. Geogr., 59, 10-21, 2007.

Walgenbach, K., Dietze, G., Hornscheidt, A. und Palm, K. (Hrsg.): Gender als interdependente Kategorie, Budrich, Opladen, 2012.

Weiß, A.: Raumrelationen als zentraler Aspekt weltweiter Ungleichheiten, Große Armut, großer Reichtum: zur Transnationalisierung sozialer Ungleichheiten, Herausgeber: Beck, U. und Poferl, A., Suhrkamp, Frankfurt/Main, 366-388, 2010.

Wichterich, C.: Geschlechteranalysen und -diskurse in der Krise, Peripherie, 118/119, 164-187, 2010.

Winker, G. und Degele, N.: Intersektionalität. Zur Analyse sozialer Ungleichheiten, transcript, Bielefeld, 2009. 